\title{
Power Consumption Optimization and Home Automaton using Smart Sensor Networks
}

\author{
Veeramanenisharath kumar, P.Anuradha
}

\begin{abstract}
The process of obtaining power consumption optimization and home automation has been accounted. We are making use of various sensors for this purpose. All the sensors used are negative coefficient sensors. Unnecessary wastage of electricity is reduced with the help of sensors. Sensors are used to get information such as light intensity,temperature, humidity, harmful gases using LDRs, thermistors, humidity sensors and gas sensors respectively. The data is given to the GSM from controller with the help of Max232 IC. With the help of GSM,one can monitor the conditions in the environment from long distance also. The model has been broadly tried, in actuality, circumstances and exploratory outcomes are exceptionally reassuring.
\end{abstract}

At first Zigbee wireless sensor network is used to monitor and control the system, but because of some limitations of the Zigbee network such as its inability for long range communications, GSM system is used in the extension part of the project.

Key Words:smart monitoring, controlling system, Zigbee wireless, GSM system.

\section{INTRODUCTION}

Various examinations disclose to us that keen homes (or) wise structures can utilize vitality more effectively than customary structures. In this manner, a few scientists have pushed building brilliant homes for lessening vitality utilization. Practically all proposed shrewd home_structures in the writing receive the remote sensor organize (WSN) as the predominant innovation. In the event that the settings of the earth can be made to react to human conduct consequently, at that point there are a few favorable circumstances. The computerization of home_settings to go about the tenant essentials is named as clever home motorization structure. Enveloping understanding responds to the direct of inhabitants in home and gives various workplaces. Standard speaking, vigilant home computerization system involves lots of sensors, gathering different sorts of info, with respect to the tenants and utility usage at home. Structures with preparing limits dismember the assimilated data to see the activities inhabitant's events.

In this present examination, the model has been generally attempted, everything considered,conditions and test outcomes are enabling. At first Zigbee remote sensor framework is used to screen and control the structure, yet since of specific hindrances of the Zigbee framework, for instance, its disappointment for long range exchanges, GSM system is used in the enlargement part of the assignment.

Revised Manuscript Received on April 12, 2019.

Veeramanenisharathkumar, PG Scholar,Department of ECE,S R Engineering college, Ananthasagar, Warangal, Telangana, India (veeramanenisharathrao001@gmail.com)

P.Anuradha, Department of ECE, S R Engineering College, Ananthasagar, Warangal, Telangana,India

\section{LITERATURE REVIEW}

Dr.S.MaryPraveena, K.Kavitha, et al.,(2017)

In this paper I utilized uninvolved infrared sensor that turn light on naturally when individual cruise by, later mood killer after a certain predefined delay for considerably more vitality saving. The vitality sparing of lighting frameworks in respect to those without shrewd control was assessed. From this investigation it was presumed that the proposed shrewd home control system utilizes the WSN for information detecting. The proposed system foundation has the benefits of WSNs and Passive infrared sensor. It improves the issue of superfluous wastage of vitality.

\section{John Batani ${ }^{1}$, Silence Dzambo ${ }^{2}$,Israel Magodi ${ }^{3}$ et al.(2017)}

The focal point of this paper was to decrease the measure of power devoured by family units, subsequently diminishing the general weight on the national power network and expanding the accessible power for modern use, prompting work creation. The proposed framework can help in the acknowledgment of the UN's SDGs through guaranteeing there is sufficient power for mechanical use, as embraced by SDG 9. From this examination it was reasoned that The Household Power Optimization and Monitoring System proposed in this concentrated for the most part on diminishing the measure of power devoured by families and consequently lessening weight on the national power network. The consequences of the framework show that the framework can diminish control utilization in family units by up to 50 percent.

\section{TECHNIQUES USED}

\section{GSM MODULE}

GSM is helpful to connect the devices to cellular services. The data is given to the GSM by the controller with the help of MAX232 IC in the form of serial communication which follows RS232 protocol. It helps to give the information of various parameters such as temperature, moisture, harmful gases to the corresponding person who wants to monitor the parameters. The mobile number of the person should be written in the program code, so that the GSM sends the information to that particular person only.

\section{SIM800L FEATURES}

It supports four bands of $850 / 900 / 1800 / 1900 \mathrm{MHz}$. SIM800 is based on a new chipset as compared to SIM900.SIM800 is definitely an improvement over 
SIM900/SIM900A as it suffers less from Modem Hang problem as compared to SIM900. One advantage is that modem are almost pin to pin compatible for most applications and follow same AT command list for common applications.

\section{BLOCK DIAGRAM}

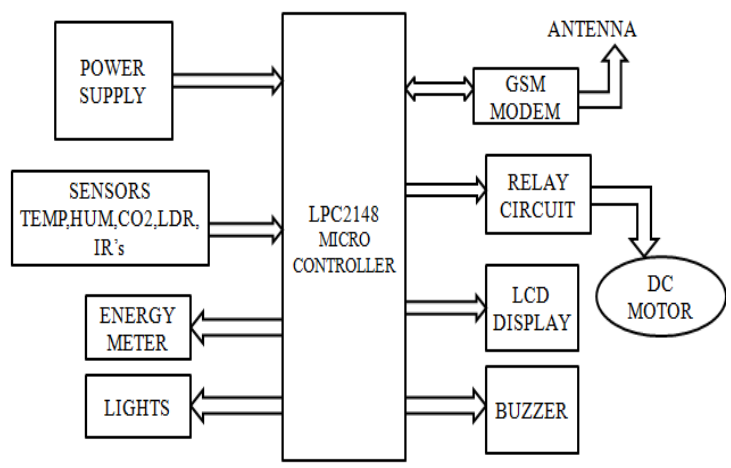

Fig1: Power consumption optimization and home automation using smart sensor networks

\subsection{Power supply}

Power supply is very important for every project. We are taking the power supply as $230 \mathrm{v}$ ac at first. We are using transformer for reducing voltage from $230 \mathrm{v}$ ac to $12 \mathrm{v}$ ac. Afterwards we are using rectifier to change $12 \mathrm{v}$ ac to $12 \mathrm{v}$ dc. We will not get pure dc with rectifier. We use filters for making fluctuating $\mathrm{dc}$ as pure dc. As most of the components use $5 \mathrm{v}$ and $3.3 \mathrm{v}$ power supply, we use 7805 voltage regulator to maintain $5 \mathrm{v}$ voltage and AMS1117 regulator to maintain $3.3 \mathrm{v}$ voltage. $\mathrm{LCD}$ wants $5 \mathrm{v}$ to operate and Controller wants $3.3 \mathrm{v}$ to operate.

\subsection{Microcontroller Section}

LPC2148 microcontroller is used in this project.LPC means low power consumption. we can say that it consumes less power because of the pipelining process used which requires less clock cycles for the execution of a program. It has ARM7 processor which has Reduced Instruction Set Architecture. This LPC2148 helps to transfer data between sensors \&GSM and it is also used for performing actions based on the signals received from various sensors.

\subsection{LCD Display Section}

This section helps to display various parameters such as temperature,gases present if any,which are poisonous, humidity and door open or close status.

\subsection{Temperature sensor}

Thermistor is used as temperature sensor in this project. Thermistor resistance differs with respect to temperature. We are using negative temperature coefficient thermistor whose resistance falls with rise in the temperature.

\subsection{Humidity sensor}

Humidity sensor is used to find the percentage of moisture in the environment. It calculates the moisture with the help of difference in the resistance when there is an increase or decrease of moisture in the environment.

\subsection{GAS sensor}

MQ3 gas sensor is used in this project.SnO2 material in MQ3 is helpful for identification of any harmful or poisonous gases. $\mathrm{SnO} 2$ material, in clean air, will have poor conductivity and it will have more conductivity if any other gases are present. so we can identify the harmful gases.

\subsection{LDR sensor}

It is a detecting gadget which is utilized to recognize the light force of the earth and dependent on that light will gleam because of that we can diminish the human assistance to switch on/off the lights.

\subsection{IR sensor \& Photo diode:}

IR sensor and photo diode is used for detecting obstacle. Photo diode is helpful to get voltage or current from the light.

\subsection{Relay Section}

This space includes of associate degree interfacing hardware to show ON/OFF the framework at no matter purpose any unfortunate conditions as an example overburden is distinguished. This hardware essentially includes of a Relay, semiconductor device associate degreed an assurance diode. A hand-off is used to drive the $230 \mathrm{~V}$ gadgets.

\subsection{Buzzer Section}

This area comprises of a Buzzer. The bell is utilized to caution/show the finishing of procedure. It is some of the time used to show the beginning of the implanted framework by alarming during start-up.

\subsection{Energy meter}

Energy meter is an electrical meter helpful to measure electrical energy made use by the consumers. It is calculated with the help of power and time. The consumers may be household, industrial, commercial etc..,.

\subsection{GSM modem}

GSM is a ton of ETSI rules deciding the structure for a mechanized cell organization. It works with RS232 interface, which grants you partner PC similarly as microcontroller with RS232 Chip(MAX232).It works with frequency 800 megahertz in portable correspondence range 800 megahertz to 1200 megahertz.

\subsection{DC motor}

DC motor is used for automatic opening and closing of the door in this project. A DC motor relies upon the manner in which that like magnet shafts repel and not in any manner like appealing posts pull in each other. A twist of wire with a present experiencing it delivers an electromagnetic field agreed with the point of convergence of the circle. By trading the current on or off in a twist its alluring field can be turned on or off or by trading the course of the current insider savvy the heading of the delivered appealing field can be traded 180 . 


\section{KEIL SOFTWARE FOR C PROGRAMMING}

The shopper of KEIL fixates on "ventures". A task may be a summary of all the supply records needed to manufacture a solitary application, all the instrument decisions that confirm exactly the way to fabricate the appliance, and - whenever needed - however the appliance got to be reenacted. A task contains enough information to require tons of supply records and make exactly the twofold code needed for the appliance. seeable of the high level of ability needed from the devices, there square measure varied alternatives which will be set to rearrange the devices to figure during a specific approach. it's dreary to wish to line these decisions up when the appliance is being fabricated; during this approach they're placed away during a task record. Stacking the task record into KEIL educates KEIL that supply documents square measure needed, wherever they are, \{and how the approach\} to style the instruments within the right way. KEIL would then be ready to execute every equipment with the correct decisions. It's in addition conceivable to create new activities in KEIL. Supply documents square measure supplemental to the enterprise and therefore the device alternatives square measure set as needed. The task would then be ready to be spared to save lots of the settings. The enterprise is reloaded and therefore take a look at system or computer program began, all the perfect windows square measure opened. KEIL enterprise records have the growth.

\section{RESULTS}

The following figures shows the output of how power consumption optimization and home automation using smart sensor networks is achieved.

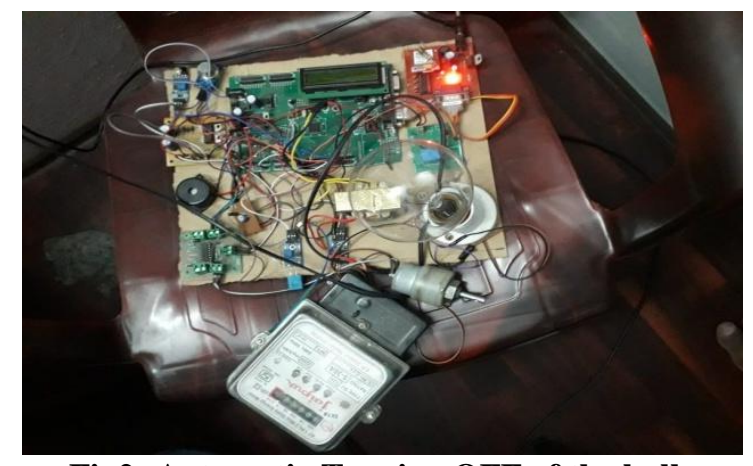

Fig2: Automatic Turning OFF of the bulb

When LDR detects the amount of light in the environment is more, then the resistance of the LDR decreases, thereby decrease in the voltage also.There is a comparator circuit provided along with the LDR circuit, which measures the difference between the reference voltage and the voltage obtained after decrease in the resistance. The reference voltage is $5 \mathrm{~V}$.The difference in voltage is given as input signal to the controller and the controller sends the signal to the relay, then the relay trips and the bulb is switched off. The above process shows clearly that the power consumption is optimized, because the bulb is turned off when there is sufficient amount of natural light in the room or any other premises, there by reducing the wastage of electricity. we can say home automation is also done in this process as the bulb is automatically turned off by the controller with the help of relay.Relay is used here because the bulb is an acapplication,so relay avoids the reverse flow of ac voltage to the controller, as the controller may get damaged.

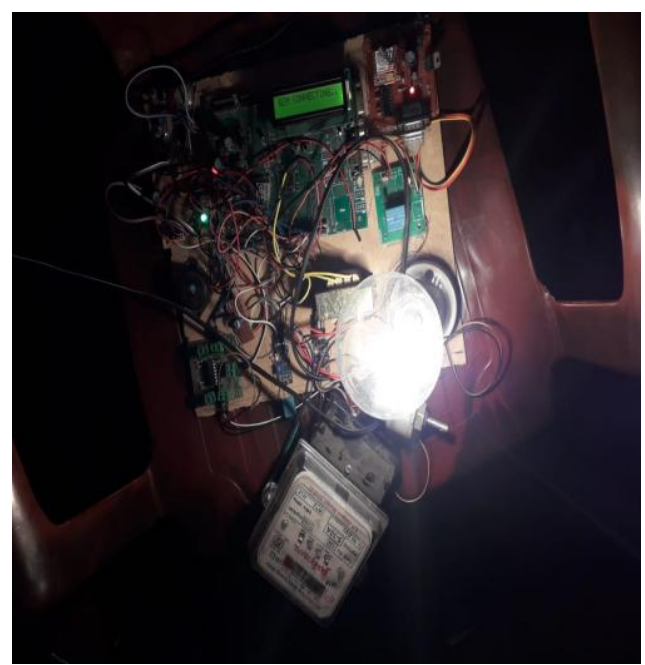

Fig 3: Automatic turning $\mathrm{ON}$ of the bulb

As the LDR detects insufficient amount of light in the premises, there will be an increase in the resistance of LDR and corresponding voltage will also increase. The reference voltage is $5 \mathrm{~V}$. The difference between the reference voltage and obtained voltage measured by comparator is given as input signal to the controller and the controller sends signal to the relay and the relay turns on the bulb automatically.Home automation is done as the bulb is automatically turned on by the controller with the help of relay, when there is no light detected by the LDR sensor.

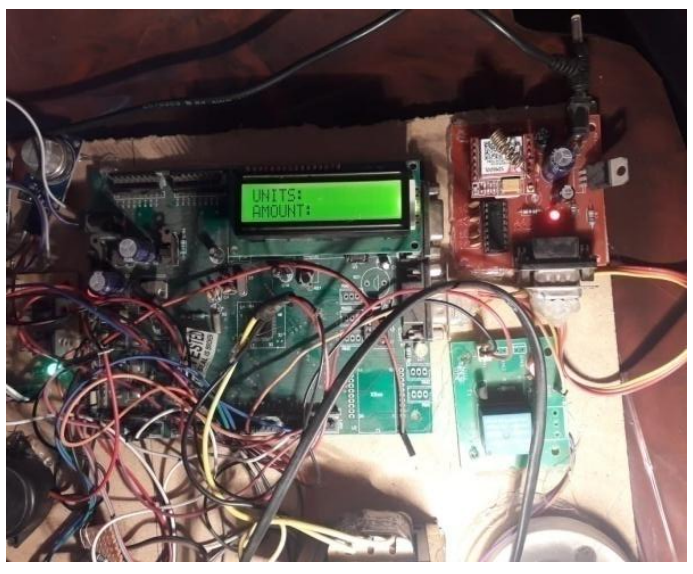

Fig 4: The initiation of measurement process

The measurement of various parameters such as temperature,gasespresent,humidity etc.., is initiated after detecting their exact amount with the help of difference in the resistance.It is measured in terms of amount and units. Blue Eyes Intelligence Engineering 


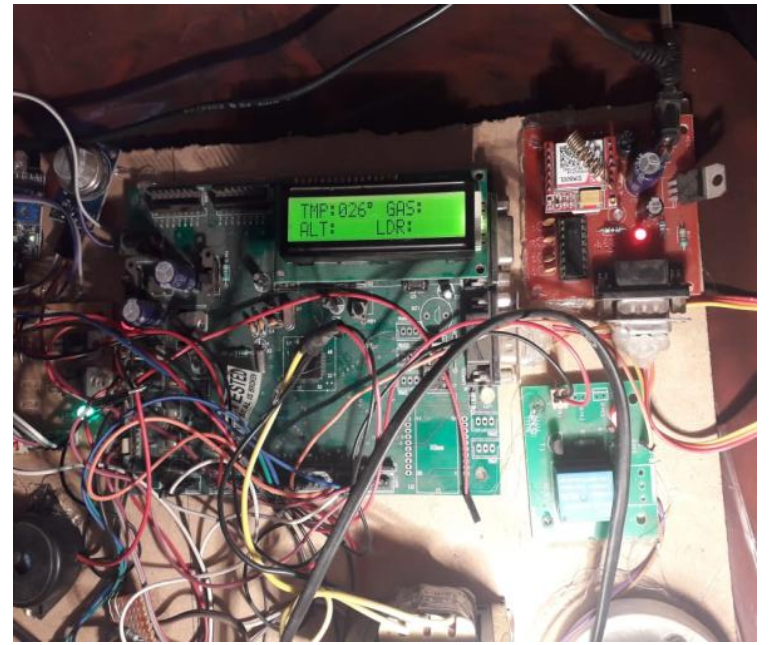

Fig 5:

Display of temperature value

It shows the display of temperature value in the LCD as $\mathbf{2 6}^{\circ} \mathbf{c}\left(\mathrm{TMP:026}^{\circ}\right)$. The temperature is measured by thermistor,as it is an instrument used for measuring temperature.It measures the temperature based on varying resistance.Along with the thermistor, a comparator circuit is provided,which measures the difference between the reference voltage and the voltage obtained because of the change in the resistance. The reference voltage is 5V.The difference in the voltage is given as input to the controller and the controller sends the information to the GSM.Home automation is done as the controller sends the information to the GSM through MAX232 IC and GSM sends the information to the corresponding person automatically, if the thermistor identifies abnormal value of temperature.

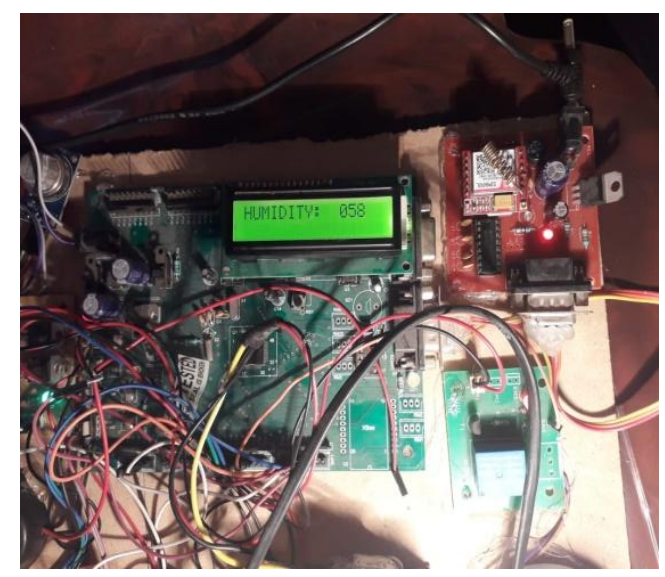

Fig 6: Display of humidity value

The value of humidity present in the environment is displayed as 58 (HUMIDITY:058) units by the humidity sensor.Along with the humidity sensor,a comparator circuit is provided,which measures the difference between the reference voltage and the voltage obtained because of the change in the resistance. The reference voltage is $5 \mathrm{~V}$. The difference in the voltage is given as input to the controller and the controller sends the information to the GSM. Home automation is done as the controller sends the information to the GSM through MAX232 IC and GSM sends the information to the corresponding person automatically, if the humidity sensor identifies abnormal value of humidity.

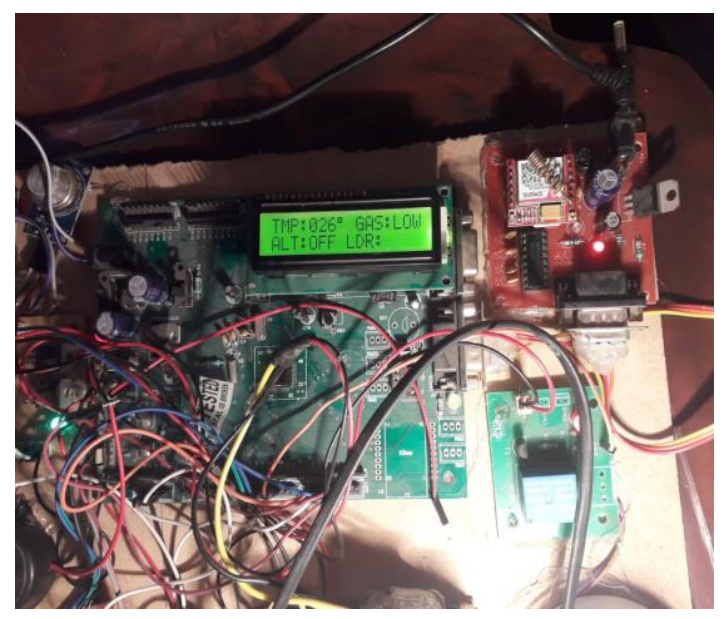

Fig 7: Display of value of temperature, gas and opening or closing condition of the door

The value of temperature is displayed as $\mathbf{2 6}^{\mathbf{}} \mathbf{c}\left(\mathbf{T M P}^{\mathrm{O}} \mathbf{0 2 6 ^ { \circ }}\right)$ in the LCD,presence of poisonous gases in the environment as low(GAS:LOW) and it also shows that the door is in the closed position (ALT:OFF)as there is no person detected by the IR sensor.There is a material called $\mathrm{SnO} 2$ wound in the gas sensor whose resistance varies with the change in the gases present in the environment. With the change in the resistance,corresponding voltage also decreases..Along with gas sensor,a comparator circuit is provided,which measures the difference between the reference voltage and the voltage obtained because of the change in the resistance. The reference voltage is $5 \mathrm{~V}$.The difference in the voltage is given as input to the controller and the controller sends the information to the GSM. Home automation is done as the controller sends the information to the GSM through MAX232 IC and GSM sends the information to the corresponding person automatically,if the thermistor,humidity sensor,gas sensor and IR sensor identifies abnormal value of temperature,humidity,harmful gases and door opening or closing condition respectively. 


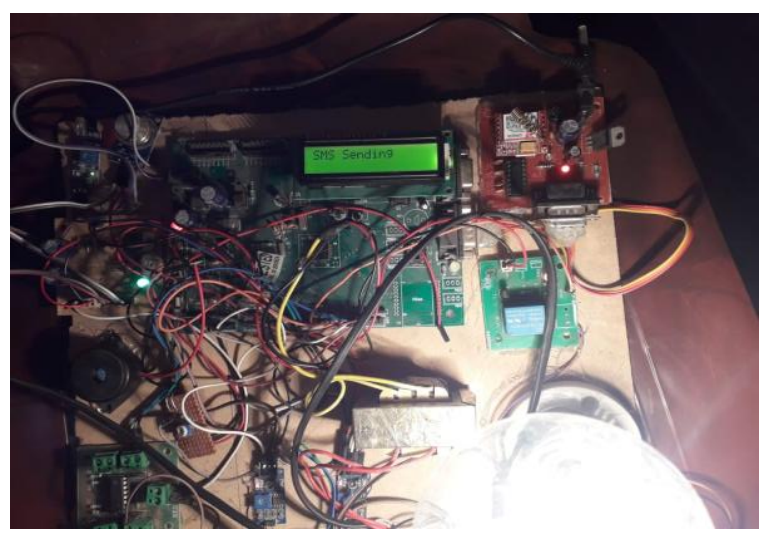

Fig 8: Display of sending of SMS alerts

The above figure shows the sending of SMS alerts(SMS SENDING) to the corresponding person who wants to know the values of temperature,amount of poisonous gases and humidity present in the environment.The Mobile number of the corresponding person is written in the program code.

\section{CONCLUSIONS}

The Household Power Optimization and home automation proposed herein focused mainly on reducing the amount of electricity consumed and wastage of electricity by households and thereby reducing stress on the national power grid.We can say that power consumption is optimized because we are using the bulb only when there is no sufficient light present in the room and if sufficient light is present,we are turning off the bulb automatically. Hence from the project it was concluded that home automation as well as power consumption optimization both are simultaneously achieved with the help of various sensors and GSM communication technology. Sensors used here are temperature sensor, light sensor, gas sensor etc.,. Motor driver circuit is also used for automatic opening and closing of the door.

\section{REFERENCES}

1. Dr.S.MaryPraveena, A.K.Kavitha and R.Kanmani, "Vitality Conservation in Smart Home Using Wireless Sensor Network", International Journal of Trend in Research and Development, Volume 4(1), ISSN: 2394-9333 www.ijtrd.com.

2. John Batani, Silence Dzambo and Israel Magodi, "Family Power Optimization and Monitoring System", International Journal of Computer Science and Business Informatics.

3. C. Molitor et al., -Multiphysics proving ground for sustainable power source frameworks in shrewd homes, IEEE Trans. Ind. Electron., vol. 60, no. 3, pp. 12351248,Mar. 2013.

4. D. M. Han and J. H. Lim, - Smart home vitality the executives framework utilizing IEEE 802.15.4 and Zigbee, || IEEE Trans. Consum. Electron., vol. 56, no. 3, pp. 14031410, Aug. 2010.

5. M. Eisenhauer, P. Rosengren, and P. Antolin, -A improvement stage for incorporating remote gadgets and sensors into encompassing knowledge systems, $\|$ in Proc. sixth
Annu. IEEE Commun. Soc. Sensor, Mesh Ad HocCommun. Netw. Workshops, Jun. 2009, pp. 1-3.

6. S. Hong, D. Kim, M. Ha, S. Bae, S. Park, W. Jung, and J. E. Kim,-SNAIL: An IP-based remote sensor system way to deal with the internetof things, \| IEEE Wireless Commun., vol. 17 , no. 6 , pp. $34-42$, Dec. 2010

7. A.I. Dounis, C. Caraiscos, "Advanced control systems engineering for energy and comfort management in a building environment A review", Renewable and sustainable Energy Reviews, vol. 13, pp. 1246-1261, 2009. 